\title{
AN EQUIVALENCE RELATION THAT IS NOT FREELY GENERATED
}

\author{
SCOT ADAMS
}

(Communicated by R. Daniel Mauldin)

\begin{abstract}
In this paper it is shown that there exists a Borel equivalence relation with countable equivalence classes that is not generated by a free Borel action of a countable discrete group.
\end{abstract}

In Feldman and Moore [1, Theorem 1, p. 291], the authors prove that any countable standard equivalence relation on a Borel space comes by taking the orbits of some Borel group acton. They then ask two questions, both relating to whether the action can be chosen to be free. (In groupoid terms, they are asking if the groupoid of such an equivalence relation is isomorphic to the groupoid coming from a group action.) The first question is Borel-theoretic, which is entirely apposite, as the theorem is of a Borel-theoretic nature. The second question is more measuretheoretic. We find a counterexample which definitively answers the first question. We show that our techniques also answer the second question in the absence of an ergodicity assumption.

One comment before starting: We prefer the terminology "Borel" to refer to an equivalence relation on a Borel space $B$ whose graph is a Borel subset of $B \times B$, as opposed to "standard" used in [1]. As in [1], an equivalence relation is called countable if all of its equivalence classes are countable.

We develop some terminology. Say $(B, R)$ is a standard countable equivalence space if $B$ is a standard Borel space and $R$ is a countable Borel equivalence relation on $B$; cf. [1, p. 291]. If $B$ is a standard Borel space and $G$ is a countable group of Borel automorphisms of $B$, then let $R_{G}$ denote the equivalence relation on $B$ defined by the orbits of the $G$-action. We then have the aforementioned theorem of Feldman and Moore [1]:

THEOREM 1. If $(B, R)$ is a standard countable equivalence space, then there exists a countable group $G$ of Borel automorphisms of $B$ such that $R=R_{G}$.

ProOF. Feldman and Moore [1, Theorem 1, p. 291]. Q.E.D.

We now refer to Zimmer [2, Definition 4.1.1, p. 59] for the definition of amenability of groups. We define amenability of equivalence relations on measure spaces as in Zimmer [3, p. 24, above Theorem 2.1]. We define $R$-invariance of a measure $\mu$ on a Borel space $B$ as in Feldman and Moore [1, p. 293]. We say that a standard countable equivalence space $(B, R)$ is $\mu$-amenable where $\mu$ is a finite, $R$-invariant measure on $B$, if $R$ is amenable on $(B, \mu)$.

Received by the editors May 12, 1987.

1980 Mathematics Subject Classification (1985 Revision). Primary 28D15.

Key words and phrases. Borel equivalence relation, measurable equivalence relation, amenable equivalence relation. 
The following is well-known.

REMARK 2. Let $(B, R)$ be a standard countable equivalence space. Let $G$ be a discrete group acting freely on $B$ through Borel automorphisms such that $R=R_{G}$. Let $\mu$ be an $R$-invariant finite measure on $B$. Then $G$ is amenable if and only if $(B, R)$ is $\mu$-amenable.

Proof. By Feldman and Moore [1, Corollary 1, p. 294], $R$-invariance of $\mu$ implies $G$-invariance of $\mu$. By Zimmer [2, Proposition 4.3.3, p. 79 and remark after Definition 4.3 .1$, p. 78], $G$ is amenable if and only if the $G$-action on $(B, \mu)$ is amenable. So it remains to show that the $G$-action on $(B, \mu)$ is amenable if and only if $(B, R)$ is $\mu$-amenable. Since the action is free, this follows immediately from the definitions. Q.E.D.

It is now easy to answer the question of Feldman and Moore mentioned at the beginning of this note. We simply choose a standard countable equivalence space $(B, R)$ which admits two finite $R$-invariant measures $\mu$ and $\nu$ such that $(B, R)$ is $\mu$-amenable, but not $\nu$-amenable. We now proceed to do this.

Let $H$ be an amenable group, e.g., let $H=\mathbf{Z}$. Let $K$ be a nonamenable group, e.g., let $K=$ the (nonabelian) free group on two letters. Let $(C, \kappa)$ be a finite measure space with a free $H$-action such that $\kappa$ is $H$-invariant. For example, let $C$ be the set of maps from $H$ into the two point Borel space with balenced probability measure (that is, with a probability measure that associates to each point the measure $1 / 2$ ). Then let $H$ act on $C$ through permutation of coordinates. Similarly, let $(D, \lambda)$ be a finite measure space with a free $K$-action such that $\lambda$ is $K$-invariant. A similar specific example works here too. Now let $B=C \amalg D, R=R_{H} \amalg R_{K}$, $\mu=\kappa \amalg 0$, and $\nu=0 \bigsqcup \lambda$. By Remark $2,(B, R)$ is $\mu$-amenable but not $\nu$-amenable. And so we can state the main conclusion of this paper:

Proposition 3. Let $(B, R)$ be defined as in the preceding paragraph. Then there is no group $G$ together with a free action on $B$ such that $R=R_{G}$.

This answers the Borel-theoretic question mentioned in the first paragraph. We now state the measure-theoretic question with ergodicity.

QUESTION 4. Let $(B, \mu)$ be a standard measure space. Let $R$ be an ergodic Borel equivalence relation on $B$ such that $\mu$ is quasi-invariant. Assume that $\mu$ a.e. $R$-equivalence class is countable. Does there necessarily exist a countable group $G$ of measure space automorphisms of $(B, \mu)$ such that $R$ is orbit equivalent to $R_{G}$ and such that the $G$-action on $B$ is free?

We conclude by remarking that in the absence of an ergodicity assumption, the answer to the above question is also no and, using the notation developed above, $(B, \mu+\nu)$ is a counterexample, by another application of Remark 2 .

\section{REFERENCES}

1. Jacob Feldman and Calvin C. Moore, Ergodic equivalence relations, cohomology and von Neumann algebras. I, Trans. Amer. Math. Soc. 234 (1977), 289-324.

2. R. J. Zimmer, Ergodic theory and semisimple groups, Birkäuser, Boston, Mass., 1984.

3. __ Hyperfinite factors and amenable ergodic actions, Invent. Math. 41 (1977), 23-31. 60637

The University of Chicago, Department of Mathematics, Chicago, Illinois 